\title{
Universiteit
}

Leiden

The Netherlands

\section{Protein energy malnutrition predicts complications in liver cirrhosis}

Huisman, E.J.; Trip, E.J.; Siersema, P.D.; Hoek, B. van; Erpecum, K.J. van

\section{Citation}

Huisman, E. J., Trip, E. J., Siersema, P. D., Hoek, B. van, \& Erpecum, K. J. van. (2011). Protein energy malnutrition predicts complications in liver cirrhosis. European Journal Of Gastroenterology And Hepatology, 23(11), 982-989. doi:10.1097/MEG.0b013e32834aa4bb

Version: $\quad$ Not Applicable (or Unknown)

License: $\quad$ Leiden University Non-exclusive license

Downloaded from: $\quad$ https://hdl.handle.net/1887/117647

Note: To cite this publication please use the final published version (if applicable). 


\section{Protein energy malnutrition predicts complications in liver cirrhosis

\author{
Ellen J. Huisman, Evelien J. Trip, Peter D. Siersema, Bart van Hoek \\ and Karel J. van Erpecum
}

\begin{abstract}
Background Protein energy malnutrition frequently occurs in liver cirrhosis. Hand-grip strength according to Jamar is most reliable to predict protein energy malnutrition. We aimed to determine whether protein energy malnutrition affects complication risk.
\end{abstract}

\begin{abstract}
Methods In 84 cirrhotics, baseline nutritional state was determined and subsequent complications prospectively assessed. Influence of potentially relevant factors including malnutrition (by Jamar hand-grip strength) on complication rates were evaluated with univariate analysis. Effect of malnutrition was subsequently evaluated by multivariate logistic regression with adjustment for possible confounders.
\end{abstract}

Results Underlying causes of cirrhosis were viral hepatitis in $31 \%$, alcohol in $26 \%$, and other in $43 \%$. Baseline Child-Pugh (CP) class was A, B, or C in 58, 35, and 7\%, respectively. Energy and protein intake decreased significantly with increasing $\mathrm{CP}$ class, with shift from proteins to carbohydrates. At baseline, according to Jamar hand-grip strength, malnutrition occurred in $67 \%(n=56)$. Malnutrition was associated with older age and higher CP class (CP class A 57\%, B 79\%, C 100\%) but not with underlying disease or comorbidity. Complications occurred in 18 and $48 \%$ in well-nourished and malnourished patients, respectively, $(P=0.007)$ during $13 \pm 6$ months follow-up. In multivariate analysis, malnutrition was an independent predictor of complications, after correcting for comorbidity, age, and CP score (adjusted odds ratio 4.230; 95\% confidence interval 1.090-16.422; $P=0.037$ ). In univariate analysis, mortality ( 4 vs. $18 \% ; P=0.1$ ) tended to be worse in malnourished patients, but this trend was lost in multivariate analysis.

Conclusion Malnutrition is an independent predictor of complications in cirrhosis. Eur J Gastroenterol Hepatol 23:982-989 (c) 2011 Wolters Kluwer Health | Lippincott Williams \& Wilkins.

European Journal of Gastroenterology \& Hepatology 2011, 23:982-989

Keywords: ascites, cirrhosis, complication, hepatic encephalopathy, hepatorenal syndrome, infection, liver, malnutrition, variceal bleeding

Departments of Gastroenterology and Hepatology, University Medical Center Utrecht and Leiden, The Netherlands

Correspondence to Karel J. van Erpecum, MD, PhD, Department of Gastroenterology and Hepatology, University Medical Center Utrecht, PO Box 85500, 3508 GA Utrecht Heidelberglaan 100, 3584 CX Utrecht, The Netherlands

Tel: + 31887555555 ; fax: + 3188 7555533; e-mail: k.j.vanerpecum@umcutrecht.nl.

Received 9 April 2011 Accepted 6 July 2011

\section{Introduction}

Protein-energy malnutrition (PEM) is a frequent phenomenon in liver cirrhosis, occurring in $20 \%$ of patients with compensated cirrhosis and in $60 \%$ or more of patients with decompensated cirrhosis [1,2]. PEM may develop in the early phase of cirrhosis [3], regardless of the underlying cause of the liver disease [4]. Its pathogenesis is multifactorial: major contributing factors are inadequate dietary intake, maldigestion, and malabsorption of both macronutrients and micronutrients and abnormal substrate utilization. In addition, reduced synthesis and increased loss of protein specifically contribute to the pathogenesis of PEM [5]. PEM is associated with impaired immunity, especially in advanced liver disease. This may increase risk of infection in this patient category with underlying intestinal bacterial overgrowth and impaired intestinal barrier function [6,7]. PEM could also increase risk of other complications, such as variceal bleeding, ascites, encephalopathy, and hepatorenal syndrome [8,9]. Furthermore, the nutritional state may affect quality of life [10] and, after liver transplantation, graft function and patient morbidity or mortality [11].

Despite its importance, PEM is often underdiagnosed in patients with cirrhosis [12], particularly in the early stages of disease [13]. Fluid retention, obesity, or other metabolic changes may interfere with diagnosing malnutrition [14]. As a result, there is no easy and decisive parameter for PEM in patients with cirrhosis [15]. Although controversial [16], hand-grip strength (HGS) according to Jamar (reflecting muscle mass and therefore protein status) is most often used to assess protein depletion in cirrhotics [17-19]. Previous studies indicated that Jamar HGS is highly sensitive but not very specific to diagnose protein depletion [15]. In this study, we aimed to (a) determine frequency of malnutrition in a group of patients with liver cirrhosis of various etiologies and in various stages of disease, with the aid of various complementary methods including Jamar HGS, and (b) 
to assess in a prospective design, the effects of nutritional state - in particular PEM - on occurrence of complications and survival in these patients.

\section{Patients and methods Patients}

In this prospective study, inclusion criteria were the presence of unequivocal cirrhosis based on a combination of clinical, laboratory, radiologic (ultrasound, MRI scan, computed tomographic scan, Fibroscan) and histologic (liver biopsy) findings and patient consent to participate in the baseline nutritional assessments and follow-up. Exclusion criteria were previous liver transplant and coexistent conditions that could affect nutritional state (e.g. gastrointestinal tract disease, malignancy, HIV positivity) and conditions interfering with determination of nutritional state (e.g. mental retardation, arthritis or other secondary diseases that could affect parameters of nutritional state such as HGS). A total of 99 consecutive patients with cirrhosis visiting the outpatient department of two University Hospitals in the period June 2007-April 2008 were considered for inclusion. Fifteen patients with cirrhosis were excluded because of baseline hepatocellular carcinoma (HCC: $n=5$ ) or pegylated-interferon-based therapy planned during follow-up $(n=10)$, considering the potential influence of these conditions on nutritional state. In the remaining 84 patients, baseline dietary history and nutritional state were determined in detail with complementary single and combined parameters. Patients visited the outpatient clinic at 6-month intervals, or more frequently if indicated. Follow-up ended in case of death, transplantation or time of final evaluation. Laboratory tests determined at baseline and at follow-up visits including liver synthetic parameters (albumin, international normalized ratio, prothrombin time), bilirubin and creatinine were determined by standard methods. Complications which had occurred before inclusion were noted. During follow-up, the following complications were registered: new onset ascites (diuretic responsive or refractory), hepatic encephalopathy, esophageal bleeding, hepatorenal syndrome, spontaneous bacterial peritonitis, other bacterial infections (like pneumonia or urinary tract infection), and HCC. The occurrence of hepatic encephalopathy was based on the Conn criteria as evaluated by the physicians in care of the patient. All complications weighed equally. Transplantation during follow-up was also recorded.

\section{Parameters of nutritional state}

Baseline dietary intake was calculated by a specialized nutritionist according to standard methods and compared with the nutritional requirements recommended for patients with liver cirrhosis in the ESPEN guidelines from 2006 [20]. Height and weight were obtained with a precision of $1 \mathrm{~cm}$ and $0.1 \mathrm{~kg}$ (Seca scale), respectively. Fluid retention, ascites, encephalopathy, and other parameters of clinical relevance were estimated by the experienced hepatologist in care for the patient. Baseline nutritional state was determined in detail with complementary single and combined parameters. Single parameters were (a) BMI corrected for fluid retention (BMIc) [21]; (b) mid-arm muscle circumference (MAMC) [22]; HGS according to (c) Jamar [17,18] or according to (d) Citec. Combined parameters were (e) body cell mass (BCM) [15] and (f) subjective global assessment (SGA) according to Hasse et al. [23]. (a) BMIc was calculated as estimated dry weight $/\left(\right.$ height) ${ }^{2}$ (in $\mathrm{kg} / \mathrm{m}^{2}$ ) [24]. BMIc cutoff values as suggested by Campillo et al. [21] were used to indicate malnutrition. These cutoff values are $22 \mathrm{~kg} / \mathrm{m}^{2}, 23 \mathrm{~kg} / \mathrm{m}^{2}$, and $25 \mathrm{~kg} / \mathrm{m}^{2}$ in patients without, with mild, and with tense ascites, respectively. (b) Mid-arm circumference (MAC) and triceps skin fold thickness (TSF) were first measured to the nearest millimeter at the nondominant arm with a measurement tape and a skin fold calliper with a pressure of $10 \mathrm{~g} / \mathrm{mm}^{2}$ of contact surface (Holtain Ltd London, UK). Measurements were taken midway between the tip of the acromion and the olecranon process, with the patient standing in a relaxed position. MAMC was then calculated from MAC and TSF with the formula MAMC $=$ MAC $-(\pi \times \mathrm{TSF})$. The average of three measurements was used. Values of MAMC were compared with those of a healthy reference population [22]. (c) Voluntary HGS was measured in the dominant hand by using a calibrated Jamar dynamometer (Biometrics, Almere, The Netherlands) adjusted for sex, age, and height and compared with a healthy reference population [18]. The best of three consecutive measurements was recorded ( $1 \mathrm{~min}$ recovery time between attempts). (d) Pinch power (Citec) was assessed with a pinch gauge (C.I.T. Technics, Centre for Innovative Technics, The Netherlands) to test the isometric muscle strength (in Newtons). Isometric strength is the torque generated by a muscle group when it is not allowed to shorten during contraction, the muscle being made to contract against an immovable load. (e) BCM: Figueiredo et al. [15] found that the combination of MAMC and HGS was the best predictor of the BCM. The combined criteria of a HGS less than $30 \mathrm{~kg}$ and a MAMC below $23 \mathrm{~cm}$ were reported to exhibit a sensitivity of $94 \%$ and a negative predictive value of $97 \%$ in identifying patients with a depleted BCM [15]. (f) The SGA adjusted for patients with liver cirrhosis by Hasse et al. [23]. This parameter comprises weight loss during previous 6 months in combination with changes in diet intake during the week before evaluation and gastrointestinal symptoms, functional capacity and fluid retention at the time point of evaluation. The physical examination focuses on loss of subcutaneous fat, muscle wasting and fluid retention. SGA is classified as normal, mild, moderate or severe malnutrition. Based on previous data [17-19], before the start of the study, we chose baseline HGS according to Jamar to distinguish well-nourished and malnourished patients. 


\section{Quality of life survey}

Quality of life was assessed using the validated Medical Outcomes Study 36-item Short-Form General Health Survey (SF-36) described in detail elsewhere [25]. The SF-36 scale does not reflect symptoms particular to certain conditions, but evaluates health comprehensively, and is used for a wide variety of disorders. SF-36 scores range from 0 (lowest) to 100 (highest), with higher scores indicating better health-related quality of life. The SF-36 is composed of 36 questions, which provide a quantitative evaluation for each of eight subscales: physical function, role-physical, bodily pain, general health perceptions, vitality, social function, role-emotional, and mental health (MH). The survey contains four domains in the area of physical health (physical function, role-physical, bodily pain, and general health perceptions) and four in the area of $\mathrm{MH}$ (vitality, social function, role-emotional, and $\mathrm{MH}$ ). Raw scores were transformed into 0-100 scales, with 0 and 100 assigned to lowest and highest possible values, respectively. Higher scores indicate better health. The scales of SF-36 were summarized into two scales: the physical component summary and the MH component summary.

\section{Statistical analysis}

Statistical analysis was carried out using SPSS version 15.0 for Windows. Data are given as mean \pm SD in case of parametric distribution and also as median and range in case of nonparametric distribution. Differences were tested for statistical analysis by dependent or independent $t$-test, Pearson $\chi^{2}$-test or analysis of variance with Fisher LSD as post-hoc test as appropriate. The Kolmogorov-Smirnov test was used to test for normal distribution. The Mann-Whitney $U$ test or the KruskalWallis test was used if the normality assumption was not met. The primary endpoints were a complication or end of follow-up.

Complications during follow-up were compared between well-nourished and malnourished groups according to Jamar HGS [17-19]. The influence of nutritional status according to Jamar HGS, age, sex, underlying cause of cirrhosis, comorbidity, CP score, and BMI score on complications and mortality during follow-up were first evaluated with univariate analysis using logistic regression. As we were interested only in the effects of nutritional state on complication risk, multivariate logistic regression was subsequently used to adjust for possible confouders: All variables with $P$-value of less than 0.200 in univariate analysis were entered in the model as covariables. Backward stepwise regression was then used to exclude variables with $P$ value of more than 0.05 . Results of logistic regression are presented as adjusted odds ratios (OR) with exact $95 \%$ confidence intervals (CI) and twosided $P$-values. Differences between Kaplan-Meier curves were tested for statistical significance using the $\log$ rank test. A two-sided $P$-value of less than 0.050 is considered statistically significant.

\section{Results}

\section{Patient characteristics}

Baseline characteristics of the 84 included patients are given in Table 1 . The underlying causes of cirrhosis were viral hepatitis in $31 \%$, alcohol in $26 \%$, and other diseases (autoimmune hepatitis, primary biliary cirrhosis, primary sclerosing cholangitis, hemochromatosis, Wilson disease) in $43 \%$ of cases. Baseline Child-Pugh (CP) class was A, B, or $\mathrm{C}$ in 58,35 , and $7 \%$, respectively. Forty-six percent of the patients exhibited significant comorbidity (diabetes mellitus, cardiovascular disease, inflammatory bowel disease). Twenty-nine percent of patients were on the waiting list for transplantation.

\section{Nutritional assessment}

Various parameters of nutritional status indicated malnutrition in $5-74 \%$ of all cases depending on the method used (Jamar 67\%, Citec 74\%, MAMC 58\%, SGA 58\%, BCM 39\%, BMIc 5\%). For most methods, malnutrition tended to be more freqent with higher CP class (Table 2). At baseline, $67 \%$ of patients $(n=56)$ were malnourished (CP class A 57\%, B 79\%, C 100\%) and 33\% ( $n=28)$ well nourished according to Jamar HGS. Of note, prevalence of obesity was high in our Dutch patients with liver cirrhosis, independent of CP class: $25 \%$ were overweight [BMI overweight (BMIc 25-29.9), 14\% were obese (BMIc 30-34.9), 5\% severely obese (BMIc 35-39.9), and $1 \%$ morbidly obese (BMIc $\geq 40$ ). Only $5 \%$ of patients were severely underweight (below $18.5 \mathrm{~kg} / \mathrm{m}^{2}$ ). BMIc, according to cutoff values of Campillo et al. [21], indicated malnutrition in $25 \%$ of cases.

At baseline assessment, energy, and protein intake were found to be significantly lower with higher CP class. Most importantly, this phenomenon was accompanied by a shift from protein to carbohydrate intake (Table 2). CP class did not significantly affect intake of vegetables, fruit or fiber. Ratio of carbohydrate to protein intake as percentage of total energy intake (energy \%) was significantly higher with more severe CP class, and was also associated with malnutrition according to Jamar HGS (Tables 1 and 2). No significant difference was found in the intake of fat (energy \%) between various CP classes or patients with or without sufficient HGS according to Jamar (Tables 1 and 2).

\section{Quality of life}

The physical and mental components of the quality of life (SF-36) tended to be lower with increasing disease severity according to CP class without reaching significance (Table 2). In malnourished patients according to Jamar HGS, the physical component of the SF-36 was significantly lower $(P<0.00001)$, whereas the mental component tended to be lower $(P=0.066$; Table 1$)$.

\section{HGS and complications}

Of the six nutritional parameters used, only insufficient Jamar HGS was an independent predictor of complications. 
Table 1 Baseline characteristics of 84 well-nourished and malnourished patients with cirrhosis based on Jamar hand-grip strength

\begin{tabular}{|c|c|c|c|c|}
\hline & All patients & Well-nourished patients & Malnourished patients & $P$ \\
\hline Number of patients & 84 & 28 & 56 & \\
\hline Duration of follow-up (months) & $13 \pm 5(1-19)$ & $14 \pm 3(7-19)$ & $12 \pm 6(1-19)$ & 0.049 \\
\hline Etiology & & & & 0.462 \\
\hline Viral hepatitis & $26(31 \%)$ & $10(36 \%)$ & $16(29 \%)$ & \\
\hline Alcoholics & $22(26 \%)$ & $5(18 \%)$ & $17(30 \%)$ & \\
\hline Other & $36(43 \%)$ & $13(46 \%)$ & $23(41 \%)$ & \\
\hline Age (years) & $55 \pm 12(22-79)$ & $51 \pm 13(23-79)$ & $56 \pm 11(22-77)$ & 0.040 \\
\hline Sex (men) & $56(67 \%)$ & $20(71 \%)$ & $36(64 \%)$ & 0.513 \\
\hline Child-Pugh class & & & & 0.047 \\
\hline A & $49(58 \%)$ & $21(75 \%)$ & $28(50 \%)$ & \\
\hline B & $29(35 \%)$ & $7(25 \%)$ & $22(39 \%)$ & \\
\hline $\mathrm{C}$ & $6(7 \%)$ & & $6(11 \%)$ & \\
\hline Comorbidity & $39(46 \%)$ & $10(36 \%)$ & $29(52 \%)$ & 0.164 \\
\hline On transplantation waiting list & $24(29 \%)$ & $5(18 \%)$ & $19(34 \%)$ & 0.075 \\
\hline \multicolumn{5}{|l|}{ Routine blood tests } \\
\hline Bilirubin $(\mu \mathrm{mol} / \mathrm{l})$ & $44 \pm 95: 24(3-845)$ & $23 \pm 14: 18(7-61)$ & $55 \pm 115: 29(3-845)$ & 0.158 \\
\hline Protrombin activity (INR) & $1.22 \pm 0.22(0.90-2.0)$ & $1.15 \pm 0.19(0.96-1.8)$ & $1.26 \pm 0.23(0.9-2.0)$ & 0.066 \\
\hline Albumin $(\mathrm{g} / \mathrm{l})$ & $35.8 \pm 6.5(20-49)$ & $37.3 \pm 5.2(23.6-47.3)$ & $34.5 \pm 6.9(20.4-49)$ & 0.065 \\
\hline Creatinine $(\mu \mathrm{mol} / \mathrm{l})$ & $88 \pm 31(43-247)$ & $82 \pm 17(52-116)$ & $91 \pm 35(43-247)$ & 0.123 \\
\hline BMlc (\% of patients with normal BMIc) ${ }^{a}$ & $25 \pm 5(16-40)(50 \%)^{a}$ & $25 \pm 4(19-37)(61 \%)^{\mathrm{a}}$ & $26 \pm 5(16-40)(45 \%)^{\mathrm{a}}$ & 0.684 \\
\hline Energy intake (kcal/day) & $2058 \pm 671(694-4916)$ & $2395 \pm 651(1181-4916)$ & $1893 \pm 622(694-3978)$ & 0.001 \\
\hline Sufficient & $59 \%$ & $74 \%$ & $51 \%$ & 0.045 \\
\hline Protein intake (g/day) & $90 \pm 31(18-206)$ & $110 \pm 29:(72-206)$ & $79 \pm 27:(18-167)$ & 0.000 \\
\hline Sufficient & $46 \%$ & $67 \%$ & $36 \%$ & 0.010 \\
\hline Energy $\%$ fat & $33 \pm 7$ & $35 \pm 7$ & $32 \pm 8$ & 0.142 \\
\hline Energy $\%$ carbohydrates & $50 \pm 9$ & $46 \pm 6$ & $52 \pm 9$ & 0.021 \\
\hline Energy \% protein & $17 \pm 4$ & $19 \pm 3$ & $16 \pm 4$ & 0.013 \\
\hline Ratio carbohydrate/protein intake (energy \%) & $3.1 \pm 1.1: 3.0(1.3-6.4)$ & $2.6 \pm 0.6: 2.5(1.5-3.8)$ & $3.4 \pm 1.2: 3.2(1.3-6.4)$ & 0.002 \\
\hline Meal (number/day) & $4.9 \pm 1.3(2-9)$ & $4.9 \pm 1.3(2.5-7.5)$ & $4.9 \pm 1.3(2-9)$ & 0.717 \\
\hline \multicolumn{5}{|l|}{ Quality of life } \\
\hline Physical component summary & $56(8-98)$ & $79(34-98)$ & $43(8-95)$ & 0.000 \\
\hline Mental component summary & $64(12-98)$ & $74(29-98)$ & $59(12-96)$ & 0.066 \\
\hline
\end{tabular}

Data are presented as $n(\%)$ or mean \pm SD (range). In case of nonparametric distribution, medians (range) are also given. $P$-values for well-nourished vs. malnourished group.

BMlc, body mass index corrected for fluid retention.

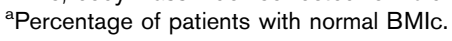

Although in univariate analysis, there was a trend for SGA and MAMC to predict complications, this trend was lost in subsequent multivariate analysis. In the well-nourished $(n=28,33 \%)$ and malnourished $(n=56,67 \%)$ groups according to Jamar HGS, follow-up was $14 \pm 3$ months and $12 \pm 6$ months, respectively $(P=0.049)$. Malnutrition according to Jamar was associated with older age and higher CP class but not with underlying disease or comorbidity (Tables 1 and 2).

Of the 24 patients on transplant waiting list at baseline, nine (38\%) were transplanted during follow-up. During follow-up, 32 patients experienced at least one new complication. At least one complication occurred in $18 \%$ and $48 \%$ of patients in the well-nourished and malnourished patients, respectively $(P=0.007)$. The malnourished group tended to experience multiple complications $(P=0.09)$. Eighteen percent of patients exhibited one complication (14 vs. $20 \%$ in well-nourished and malnourished groups), $10 \%$ of patients exhibited two complications (4 vs. $13 \%$ in well-nourished and malnourished groups), $4 \%$ of patients exhibited three complications ( 0 vs. $5 \%$ in well-nourished and malnourished groups), $5 \%$ of patients exhibited four complications ( 0 vs. $7 \%$ in well-nourished and malnourished groups), and $2 \%$ of patients exhibited five complications ( 0 vs. $3 \%$ in well-nourished and malnourished groups). Individual complications are given in Table 3.
In univariate analysis using logistic regression, malnutrition measured with HGS according to Jamar (OR 4.3; CI: 1.4-12.9), CP score (OR 2.0; CI: 1.5-2.9), age (OR 1.03; CI: $0.99-1.07$ ), and comorbidity (OR 0.56; CI: $0.23-1.37$ ) were variables with $P$-value $<0.2$ when comparing patients with and without complications during follow-up (Table 4). In multivariate analysis with backward stepwise logistic regression, malnutrition was an independent predictor of complications, after correcting for age, comorbidity, and CP score (adjusted OR 4.230; 95\% CI: 1.09-16.4; $P=0.037$ ). Sensitivity, specificity, positive, and negative predicted values of insufficient HGS to predict complications in our cirrhotic group were $84,44,48$, and $82 \%$, respectively. When comparing complication rates during the entire follow-up in well versus malnourished patients for all CP classes, the two curves were significantly different (log rank test, $P=0.003$; Fig. 1) and for the subgroup of patients with CP class A compensated cirrhosis (log rank test, $P=0.016$ ), whereas differences did not reach statistical significance in the subgroups with $\mathrm{CP}$ class B or C cirrhosis.

Mortality tended to be higher in the malnourished group (4 vs. $18 \%$ in the well-nourished and malnourished groups; $P=0.067)$. A Kaplan-Meier curve revealed a nearly significant difference between the survival of wellnourished and malnourished groups $(P=0.056, \log$ rank test). In univariate analysis, malnourishment tended to 
Table 2 Nutritional intake and state in patients with cirrhosis according to Child-Pugh class

\begin{tabular}{|c|c|c|c|c|}
\hline & Child-Pugh class $\mathrm{A}$ & Child-Pugh class B & Child-Pugh class $\mathrm{C}$ & $P$-value (two-sided) \\
\hline Number & 49 & 29 & 6 & \\
\hline \multicolumn{5}{|l|}{ HGS } \\
\hline Jamar sufficient & $21(43 \%)$ & $7(21 \%)$ & $0(0 \%)$ & $0.047 * * * * * * *$ \\
\hline Citec sufficient & $15(30 \%)$ & $6(21 \%)$ & $1(17 \%)$ & 0.563 \\
\hline MAMC sufficient & $21(43 \%)$ & $13(45 \%)$ & $1(17 \%)$ & 0.541 \\
\hline SGA sufficient & $28(57 \%)$ & $7(24 \%)$ & $0(0 \%)$ & $0.002 *, * *, * * *$ \\
\hline BCM sufficient & $32(67 \%)$ & $17(61 \%)$ & 2 (33\%) & 0.278 \\
\hline BMlc (normal) ${ }^{\mathrm{a}}$ & $25 \pm 4(18-37)(53 \%)^{a}$ & $26 \pm 6(16-40)(45 \%)^{a}$ & $24 \pm 5(16-32)(50 \%)^{a}$ & 0.311 \\
\hline Energy intake (kcal) & $2200 \pm 705(833-4916)$ & $1982 \pm 461(1087-2712)$ & $1245 \pm 630(694-2133)$ & $0.003 * *, * * *$ \\
\hline Sufficient & $74 \%$ & $38 \%$ & $17 \%$ & $0.002 *, * *, * * *$ \\
\hline Protein intake $(\mathrm{g})$ & $97 \pm 32(18-206)$ & $81 \pm 27(37-141)$ & $59 \pm 18(38-77)$ & $0.014^{*} * * *$ \\
\hline Sufficient & $59 \%$ & $28 \%$ & 0 & $0.005 *, * * * * *$ \\
\hline Energy $\%$ fat & $34 \pm 7$ & $32 \pm 8$ & $32 \pm 7$ & 0.522 \\
\hline Energy \% carbohydrates & $48 \pm 8$ & $52 \pm 9$ & $52 \pm 11$ & 0.238 \\
\hline Energy $\%$ protein & $18 \pm 3$ & $16 \pm 4$ & $16 \pm 4$ & 0.232 \\
\hline Ratio carbohydrate/protein intake (energy \%) & $2.9 \pm 0.93(1.3-6.1)$ & $3.4 \pm 1.2(1.8-6.4)$ & $3.9 \pm 1.4(1.9-5.0)$ & $0.023 *$ \\
\hline Fruit intake (pieces) & $1.8 \pm 1.7(0-10)$ & $1.5 \pm 1.2(0-5.5)$ & $1.5 \pm 1.0(0-3)$ & 0.706 \\
\hline Sufficient & $47 \%$ & $44 \%$ & $20 \%$ & 0.544 \\
\hline Vegetable intake $(\mathrm{g})$ & $106 \pm 66(0-270)$ & $98 \pm 76(0-300)$ & $30 \pm 23(0-50)$ & 0.106 \\
\hline Sufficient & $25 \%$ & $22 \%$ & $20 \%$ & 0.946 \\
\hline Fiber $(\mathrm{g})$ & $25 \pm 11(8-64)$ & $21 \pm 7(8-40)$ & $15 \pm 9(8-28)$ & 0.114 \\
\hline Sufficient & $42 \%$ & $22 \%$ & $25 \%$ & 0.216 \\
\hline Meal (number/day) & $4.8 \pm 1.3(2-8)$ & $5.2 \pm 1.5(3-9)$ & $5.1 \pm 1.5(3-9)$ & 0.297 \\
\hline \multicolumn{5}{|l|}{ Quality of life } \\
\hline Physical component summary & $60(9-98)$ & $54(8-96)$ & $34(12-96)$ & 0.194 \\
\hline Mental component summary & $66(23-97)$ & $56(15-98)$ & $43(12-96)$ & 0.257 \\
\hline
\end{tabular}

Data are presented as $n(\%)$ or mean \pm SD (range). P-value is calculated with one-way analysis of variance, Pearson $\chi^{2}$ or Kruskall-Wallis test. With post-hoc test (Fisher LSD).

BCM, body cell mass; BMlc, body mass index corrected for fluid retention; HGS, hand-grip strength; MAMC, mid-arm muscle circumference; SGA, subjective global assessment.

*Significant difference $(P<0.05)$ for $\mathrm{CP}$ class A vs. B.

***For B vs. C.

****For A vs. C.

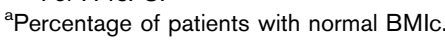

Table 3 Complications and mortality during follow-up of 84 well-nourished and malnourished patients with cirrhosis based on Jamar handgrip strength

\begin{tabular}{|c|c|c|c|c|}
\hline & \multirow[b]{2}{*}{ All patients } & \multicolumn{3}{|c|}{ According to Jamar hand-grip strength } \\
\hline & & Well-nourished patients & Malnourished patients & $P$ \\
\hline Number & 84 & 28 & 56 & \\
\hline Duration of follow-up (months) & $13 \pm 5(1-19)$ & $14 \pm 3(7-19)$ & $12 \pm 6(1-19)$ & 0.049 \\
\hline Complications at follow-up & $32(38 \%)$ & $5(18 \%)$ & $27(48 \%)$ & 0.007 \\
\hline New onset ascites & $20(24 \%)$ & $5(18 \%)$ & $15(27 \%)$ & 0.365 \\
\hline Hepatic encephalopathy & $16(19 \%)$ & 0 & $16(29 \%)$ & 0.000 \\
\hline Esophageal bleeding & $6(7 \%)$ & 0 & $6(11 \%)$ & 0.072 \\
\hline Hepatorenal syndrome & $7(8 \%)$ & 0 & $7(13 \%)$ & 0.051 \\
\hline Spontaneous bacterial peritonitis & $8(10 \%)$ & 0 & $8(14 \%)$ & 0.035 \\
\hline Other bacterial infections & $11(13 \%)$ & $1(4 \%)$ & $10(18 \%)$ & 0.067 \\
\hline Hepatocellular carcinoma & $4(5 \%)$ & $1(4 \%)$ & $3(5 \%)$ & 0.717 \\
\hline Mortality & $11(13 \%)$ & $1(4 \%)$ & $10(18 \%)$ & 0.067 \\
\hline
\end{tabular}

Data are presented as $n(\%)$ or mean \pm SD (range).

be associated with mortality $(P=0.1)$, but this trend was lost in subsequent multivariate analysis.

Laboratory values at baseline and at end of follow-up in the well-nourished and malnourished groups are given in Table 5. Serum albumin levels decreased significantly in both groups, whereas serum creatinine levels increased significantly only in the malnourished group. At end of follow-up, serum creatinine increased significantly more from baseline values in the malnourished than in the wellnourished group (increase +2.39 vs. $+19.92 \mu \mathrm{mol} / \mathrm{l}$; $P=0.04$ ). Changes of serum bilirubin (increase at end of follow-up from basal $+6.19 \mu \mathrm{mol} / \mathrm{l}$ and $+12.84 \mu \mathrm{mol} / \mathrm{l}$ in well-nourished and malnourished groups, respectively), albumin (decrease -1.13 and $-2.17 \mathrm{~g} / \mathrm{l}$, respectively), and prothrombin time (change from basal -0.04 and 0.23 , respectively) did not differ significantly between well-nourished and malnourished groups.

\section{Discussion}

The main findings of this prospective study can be summarized as follows: (a) there is a high prevalence of malnutrition (25-70\% depending on method of evaluation) in patients with liver cirrhosis in the Netherlands, 
Table 4 Univariate analysis of various variables on occurrence of new complications during follow-up in $\mathbf{8 4}$ patients with cirrhosis

\begin{tabular}{llllllll}
\hline & \multicolumn{3}{c}{ New complications } & & \multicolumn{3}{c}{ Mortality } \\
\cline { 2 - 3 } \cline { 7 - 8 } & OR & $95 \% \mathrm{Cl}$ & $P$ & & OR & $95 \% \mathrm{Cl}$ & $P$ \\
\hline Hand-grip strength* & 4.3 & $1.4-12.9$ & 0.010 & & 5.87 & $0.71-48.4$ & 0.100 \\
Age & 1.03 & $0.99-1.07$ & 0.169 & & 1.08 & $1.01-1.16$ & 0.022 \\
Sex & 1.88 & $0.71-4.98$ & 0.207 & & 1.22 & $0.28-5.28$ & 0.795 \\
Etiology & 0.78 & $0.46-1.29$ & 0.316 & & 0.72 & $0.34-1.51$ & 0.384 \\
Comorbidity & 0.56 & $0.23-1.37$ & 0.200 & & 2.24 & $0.6-8.33$ & 0.228 \\
CP score & 2.04 & $1.45-2.86$ & 0.000 & & 2.07 & $1.38-3.1$ & 0.000 \\
BMlc & 1.00 & $0.91-1.10$ & 0.954 & & 1.025 & $0.91-1.16$ & 0.691
\end{tabular}

BMlc, body mass index corrected for fluid retention; $95 \% \mathrm{Cl}, 95 \%$ confidence interval; CP, Child-Pugh; OR, odds ratio.

*According to Jamar.

Fig. 1

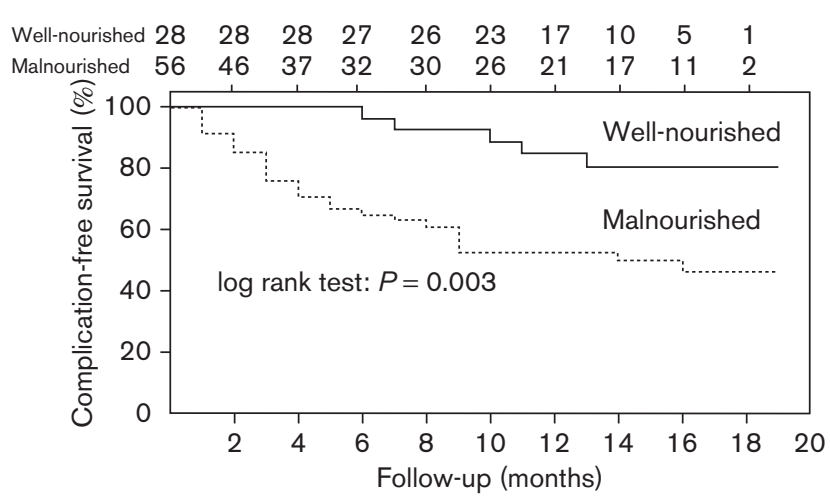

Complication-free survival is significantly lower in malnourished than in well-nourished patients with cirrhosis according to Jamar hand-grip strength test (log rank test, $P=0.003$ ).

even in early stages of the disease; (b) risk of complications is significantly increased in case of malnutrition; (c) intake of both energy and protein decreases progressively with increasing disease severity according to CP class and is associated with PEM. We evaluated PEM particularly with Jamar HGS. Jamar HGS could be affected by polyneuropathy, especially in alcoholic and diabetic patients with cirrhosis. Although not significant, patients with diabetes (11 of 13 patients) and alcoholic cirrhosis (17 of 22 patients) tended to be overrepresented in the malnourished group. Nevertheless, malnutrition according to Jamar was associated with older age and higher CP class but not with cause of underlying liver disease or comorbidity (Tables 1 and 2). In addition, Jamar HGS was an independent prognostic factor for complications during follow-up in multivariate analysis, after adjusting for several factors including comorbidity. In line with other studies [19], Jamar HGS proved to be superior to other anthropometric parameters of nutritional state to predict complications. Differences in complication rates between well-nourished and malnourished patients were significant for the subgroup of patients with CP class A compensated cirrhosis, but not for the subgroups with CP class $\mathrm{B}$ or $\mathrm{C}$ cirrhosis. This phenomenon is not unexpected: in patients with $\mathrm{CP}$ class $\mathrm{B}$ or $\mathrm{C}$ decompensated cirrhosis, other factors than malnutrition such as presence of ascites, bacterial overgrowth, and impaired defence mechanisms against infection are probably predominant in determining complication risk. In addition, the underlying mechanisms for the association between nutritional state and certain complications such as bacterial infections seem evident, whereas this is not entirely clear for other complications such as variceal bleeding. The prevalence of PEM among our Dutch patients with decompensated cirrhosis in our study is very similar to previous data from other countries [26]. Of note, a significant proportion of our patients with compensated cirrhosis also exhibited malnutrition, suggesting nutritional deficiency in relatively early stages of disease. Of special note, prevalence of overweight and obesity (after correction for ascites, BMIc) were high in our Dutch patients with liver cirrhosis, and independent of CP class. Overweight and obesity are known risk factors for development of cirrhosis [27]. Severe underweight was found in only $5 \%$ of Dutch patients, also independent of CP class. The physical and mental components of the quality of life (SF-36) tended to be lower with increasing disease severity according to CP class, without reaching significance. In contrast, the physical component of the SF-36 was remarkably and highly significantly lower in the malnourished patients according to Jamar HGS. This suggests that malnutrition according to Jamar HGS is associated with excessive overall physical discomfort. The mental component of the SF-36 also tended to be lower in the malnourished group.

The decrease of energy and protein intake with increasing CP class and in HGS insufficiency coincided with a shift from protein to carbohydrate as reflected by increasing ratio of carbohydrate/protein intake (energy $\%)$. In contrast, fat intake (energy \%) did not change (see Tables 1 and 2). Despite the decrease in energy intake, the BMIc did not differ between patients in the three CP classes or between patients with sufficient or insufficient HGS. Together, these findings suggest that inadequate protein intake could explain, at least partly, the high prevalence of PEM in our patients.

Our findings would suggest that dietary interventions could improve PEM and might reduce complication rate. Our findings also suggest that protein enrichment rather than increased calorie intake per se should be emphasized when counseling a patient with cirrhosis. Similarly, the high prevalence of overweight we found, combined with a high prevalence of insulin resistance found in general in cirrhotics [28], indicates that only sufficient intake of carbohydrates with a low glycemic load is wise in most patients. It also leads us to suggest that protein malnutrition (PM) rather than PEM could describe the 
Table 5 Laboratory measurements at baseline and end of follow-up in 84 well-nourished and malnourished patients with cirrhosis based on Jamar hand-grip strength

\begin{tabular}{lccc}
\hline & Baseline & End of follow-up & $P$ \\
\hline Well-nourished patients $(n=28)$ & & & \\
$\quad$ Bilirubin $(\mu \mathrm{mol} / \mathrm{l})$ & $23 \pm 14: 18(7-61)$ & $1.14 \pm 0.14(0.95-1.41)$ & 0.220 \\
Protrombin activity $(\mathrm{INR})$ & $1.15 \pm 0.19(0.96-1.8)$ & $36.1 \pm 5.2(24.9-45)$ & 0.166 \\
Albumin $(\mathrm{g} / \mathrm{l})$ & $37.3 \pm 5.2(23.6-47.3)$ & $85 \pm 19(47-114)$ & 0.039 \\
$\quad$ Creatinine $(\mu \mathrm{mol} / \mathrm{l})$ & $82 \pm 17(52-116)$ & $69 \pm 127: 23(5-544)$ & 0.333 \\
Malnourished patients $(n=56)$ & $55 \pm 115: 29(3-845)$ & $1.47 \pm 1.23(0.89-8.6)$ & 0.236 \\
$\quad$ Bilirubine $(\mu \mathrm{mol} / \mathrm{l})$ & $1.26 \pm 0.23(0.9-2)$ & $32.2 \pm 7.8(13-46)$ & 0.037 \\
Protrombin activity $(\mathrm{INR})$ & $34.5 \pm 6.9(20.4-49)$ & $107 \pm 68: 93(44-402)$ & 0.020 \\
Albumine $(\mathrm{g} / \mathrm{l})$ & $91 \pm 35: 81.5(43-247)$ & &
\end{tabular}

Data are presented as mean $\pm \mathrm{SD}$ (range). In case of nonparametric distribution, medians are also given.

nutritional state in most patients with cirrhosis. Indeed, nutritional interventions with protein-enriched supplements have been shown to improve nutritional status and nitrogen balance in cirrhotics [29,30]. Especially lateevening supplements seem to be effective: in a recently reported controlled trial, patients with cirrhosis were randomized to either daytime or late evening administration supplements (providing $26 \mathrm{~g}$ protein). There was a highly significant improvement of total body protein status in the late evening group, which was not the case in the daytime group [29]. These interventions may improve surrogate variables such as $\mathrm{CP}$ score, serum albumin, and serum bilirubin and possibly mortality as the most relevant clinical endpoint in patients with cirrhosis [31]. Indeed, preoperative nutritional intervention has been reported to reduce infection rates and length of hospital stay after liver transplantation [32]. Quality of life may be influenced positively by nutritional intervention as well [10]. It should also be realized that nutrient intake and nutritional patterns play a key role in genesis and progression of specific liver diseases (e.g. alcoholic liver injury) [33]. Nevertheless, it should be realized, that no prospective trials have unequivocally demonstrated that nutritional intervention and proteinenriched supplements could improve the course of cirrhotic liver disease. In univariate analysis, malnourishment tended to be associated with mortality $(P=0.1)$, but this trend was lost in subsequent multivariate analysis (see Results section). Apparently, malnourishment was not an independent factor for mortality in this study. This could relate to the possibility that other factors (e.g. CP score) might be more prominent factors for mortality and/or to the possibility that this study was underpowered to detect an independent effect of malnutrition on mortality.

Nevertheless, factors other than nutritional intake may affect nutritional status in patients with cirrhosis as well. Albumin synthesis seems to parallel liver function, that is the more compromised the liver the less the albumin production rate. Nevertheless, meal-induced albumin synthesis is impaired even in compensated patients with cirrhosis. Skeletal muscle protein synthesis is diminished in cirrhosis and total muscle protein breakdown seems to be increased, thus explaining the reduced muscle mass. Specific degradation of myofibrillar protein may lead to decreased muscle function as well [34,35]. Either hormone or substrate resistance may be involved and substances such as cytokines, insulin-like growth factor 1 , or leptin may play a role in the reduced synthesis of both albumin and muscle proteins in liver cirrhosis [36]. Finally, the physical inactivity associated with severe liver disease may also contribute to muscle wasting. It also remains to be seen whether decreased HGS according to Jamar signifies PM per se or is a more general parameter for the severity of disease. In conclusion, we found a high prevalence of PM in patients with cirrhosis even in the early stages of disease. The prevalence of (often severe) overweight was also high. PEM, as assessed by HGS according to Jamar, was an independent predictor of complications. Whether dietary counseling could reduce PEM, overweight, and the high complication risk associated with these conditions remains to be seen.

\section{Acknowledgements}

This study was supported by an unrestricted grant of Schering Plough (Houten, The Netherlands).

\section{Conflicts of interest}

K.J. van Erpecum served as an advisory board member for Gilead and Bristol Meyers Squibb. B. van Hoek served as an advisory Board Member for Janssen, Bristol Meyers Squibb, and Merck Sharp \& Dohme.

\section{References}

1 Kalaitzakis E, Simren M, Olsson R, Henfridsson P, Hugosson I, Bengtsson $\mathrm{M}$, et al. Gastrointestinal symptoms in patients with liver cirrhosis: associations with nutritional status and health-related quality of life. Scand J Gastroenterol 2006; 41:1464-1472.

2 Guglielmi FW, Panella C, Buda A, Budillon G, Caregaro L, Clerici C, et al. Nutritional state and energy balance in cirrhotic patients with or without hypermetabolism. Multicentre prospective study by the 'Nutritional Problems in Gastroenterology'. Section of the Italian Society of Gastroenterology (SIGE). Dig Liver Dis 2005; 37:681-688.

3 Cabre E, Gassull MA. Nutrition in liver disease. Curr Opin Clin Nutr Metab Care 2005; 8:545-551.

4 Thuluvath PJ, Triger DR. Evaluation of nutritional status by using anthropometry in adults with alcoholic and nonalcoholic liver disease. Am J Clin Nutr 1994; 60:269-273. 
5 O'Brien A, Williams R. Nutrition in end-stage liver disease: principles and practice. Gastroenterology 2008; 134:1729-1740.

6 Detsky AS, Smalley PS, Chang J. The rational clinical examination Is this patient malnourished? JAMA 1994; 271:54-58.

7 Zhang W, Gu Y, Chen Y, Deng H, Chen L, Chen S, et al. Intestinal flora imbalance results in altered bacterial translocation and liver function in rats with experimental cirrhosis. Eur J Gastroenterol Hepatol 2010; 22:1481-1486.

8 Alberino F, Gatta A, Amodio P, Merkel C, Di PL, Boffo G, et al. Nutrition and survival in patients with liver cirrhosis. Nutrition 2001; 17:445-450.

9 Kondrup J. Nutrition in end stage liver disease. Best Pract Res Clin Gastroenterol 2006; 20:547-560.

10 Norman K, Kirchner H, Lochs H, Pirlich M. Malnutrition affects quality of life in gastroenterology patients. World J Gastroenterol 2006; 12:3380-3385.

11 McCullough AJ. Malnutrition in liver disease. Liver Transp/ 2000; 6:S85-S96.

12 Cabre E, Gassull MA. Nutrition in chronic liver disease and liver transplantation. Curr Opin Clin Nutr Metab Care 1998; 1:423-430.

13 Roongpisuthipong C, Sobhonslidsuk A, Nantiruj K, Songchitsomboon S. Nutritional assessment in various stages of liver cirrhosis. Nutrition 2001; 17:761-765.

14 Muller MJ, Lautz HU, Plogmann B, Burger M, Korber J, Schmidt FW. Energy expenditure and substrate oxidation in patients with cirrhosis: the impact of cause, clinical staging and nutritional state. Hepatology 1992; 15:782-794.

15 Figueiredo FA, Dickson ER, Pasha TM, Porayko MK, Therneau TM, Malinchoc $\mathrm{M}$, et al. Utility of standard nutritional parameters in detecting body cell mass depletion in patients with end-stage liver disease. Liver Transp/ 2000; 6:575-581.

16 Watters DA, Haffejee AA, Angorn IB, Duffy KJ. Nutritional assessment by hand grip dynamometry. S Afr Med J 1985; 68:585-587.

17 Mathiowetz V, Weber K, Volland G, Kashman N. Reliability and validity of grip and pinch strength evaluations. J Hand Surg Am 1984; 9:222-226.

18 Webb AR, Newman LA, Taylor M, Keogh JB. Hand grip dynamometry as a predictor of postoperative complications reappraisal using age standardized grip strengths. J Parenter Enteral Nutr 1989; 13:30-33.

19 Alvares da Silva MR, Reverbel da Silveira T. Comparison between handgrip strength, subjective global assessment, and prognostic nutritional index in assessing malnutrition and predicting clinical outcome in cirrhotic outpatients. Nutrition 2005; 21:113-117.

20 Plauth M, Cabre E, Riggio O, Assis-Camilo M, Pirlich M, Kondrup J, et al. ESPEN guidelines on enteral nutrition: liver disease. Clin Nutr 2006; 25:285-294.

21 Campillo B, Richardet JP, Bories PN. Validation of body mass index for the diagnosis of malnutrition in patients with liver cirrhosis. Gastroenterol Clin Biol 2006; 30:1137-1143.
22 Frisancho AR. New standards of weight and body composition by frame size and height for assessment of nutritional status of adults and the elderly. $A m$ J Clin Nutr 1984; 40:808-819.

23 Hasse J, Strong S, Gorman MA, Liepa G. Subjective global assessment: alternative nutrition-assessment technique for liver-transplant candidates. Nutrition 1993; 9:339-343.

24 Richards J, Gunson B, Johnson J, Neuberger J. Weight gain and obesity after liver transplantation. Transpl Int 2005; 18:461-466.

25 Ware JE Jr., Sherbourne CD. The MOS 36-item short-form health survey (SF-36). I. Conceptual framework and item selection. Med Care 1992; 30:473-483.

26 Nutritional status in cirrhosis. Italian Multicentre Cooperative Project on Nutrition in Liver Cirrhosis. J Hepatol 1994; 21:317-325.

27 Hessheimer AJ, Forner A, Varela M, Bruix J. Metabolic risk factors are a major comorbidity in patients with cirrhosis independent of the presence of hepatocellular carcinoma. Eur J Gastroenterol Hepatol 2010; 22 1239-1244.

28 Goral V, Atalay R, Kucukoren M. Insulin resistance in liver cirrhosis. Hepatogastroenterology 2010; 57:309-315.

29 Plank LD, Gane EJ, Peng S, Muthu C, Mathur S, Gillanders L, et al. Nocturnal nutritional supplementation improves total body protein status of patients with liver cirrhosis: a randomized 12-month trial. Hepatology 2008; 48:557-566.

30 Verboeket-van de Venne WP, Westerterp KR, van Hoek B, Swart GR. Energy expenditure and substrate metabolism in patients with cirrhosis of the liver: effects of the pattern of food intake. Gut 1995; 36:110-116.

31 Cabre E, Gonzalez-Huix F, Bad-Lacruz A, Esteve M, Acero D, FernandezBanares $F$, et al. Effect of total enteral nutrition on the short-term outcome of severely malnourished cirrhotics. A randomized controlled trial. Gastroenterology 1990; 98:715-720.

32 Nickkholgh A, Schneider H, Encke J, Buchler MW, Schmidt J, Schemmer P. PROUD: Effects of preoperative long-term immunonutrition in patients listed for liver transplantation. Trials 2007; 8:20.

33 Gramenzi A, Caputo F, Biselli M, Kuria F, Loggi E, Andreone P, et al. Review article: alcoholic liver disease - pathophysiological aspects and risk factors. Aliment Pharmacol Ther 2006; 24:1151-1161.

34 Morrison WL, Bouchier IA, Gibson JN, Rennie MJ. Skeletal muscle and whole-body protein turnover in cirrhosis. Clin Sci (Lond) 1990; 78: 613-619.

35 Zoli M, Marchesini G, Dondi C, Bianchi GP, Pisi E. Myofibrillar protein catabolic rates in cirrhotic patients with and without muscle wasting. Clin Sci (Lond) 1982; 62:683-686.

36 Tessari P. Protein metabolism in liver cirrhosis: from albumin to muscle myofibrils. Curr Opin Clin Nutr Metab Care 2003; 6:79-85. 